\title{
Research of Sands Clogging by Microbial-Induced
}

\author{
Huang Shaoqi ${ }^{1, ~ *}$, Qian Lixi ${ }^{1}$, Lu Taishan ${ }^{1}$, Wang Xiangyang ${ }^{2}$, Shen Junqian ${ }^{3}$ \\ ${ }^{1}$ School of Transportation, Southeast University, Nanjing, China \\ ${ }^{2}$ School of Biological Science \& Medical Engineering, Southeast University, Nanjing, China \\ ${ }^{3}$ School of Civil Engineering, Southeast University, Nanjing, China \\ Email address: \\ 213141481@seu.edu.cn (Huang Shaoqi) \\ ${ }^{*}$ Corresponding author
}

\section{To cite this article:}

Huang Shaoqi, Qian Lixi, Lu Taishan, Wang Xiangyang, Shen Junqian. Research of Sands Clogging by Microbial-Induced. Science Discovery. Vol. 5, No. 3, 2017, pp. 223-228. doi: 10.11648/j.sd.20170503.21

Received: February 3, 2017; Accepted: March 1, 2017; Published: May 11, 2017

\begin{abstract}
Experiments are designed to study the effect of soil clogging by microbial-induced calcite precipitation in the short term, and the differences clogging effect under different nutrient solution. Based on the constant head permeability test, microbial-induced calcite precipitation were performed in 2 groups of sand columns, where potato soup and nutrient solution (potato- $\mathrm{Co}\left(\mathrm{NH}_{2}\right)_{2}-\mathrm{CaCl}_{2}$ ) were added. Changing trends of the hydraulic conductivity were presented by measuring piezometer readings regularly and soil micro-pore structure change was observed by scanning electron microscopy. Results show that clogging effects can be received for supply of potato soup and nutrient solution in order for the growth of microorganisms. The hydraulic conductivity of sand columns added potato soup could be reduced by $83.64 \%$ in 10 days, while that of sand columns added nutrient solution could be down to $99.14 \%$. A piece of membrane material was observed, which made soil immersed in nutrient solution bond together. Few changes took place at the surface of soil that was immersed in potato soup and the soil was still in the decentralized state. Tests showed that microbial plugging technology is able to improve soil properties in a short time, achieve effective clogging, and clogging effect remains stable within a certain time.
\end{abstract}

Keywords: Sands, Microbial-Induced Calcite Precipitation, Clogging, Modified

\section{砂土中微生物封堵机理的研究}

黄少琦 ${ }^{*}$, 钱李希 ${ }^{1}$, 鲁泰山 ${ }^{1}$, 王向阳 ${ }^{2}$, 沈君乾 ${ }^{3}$

${ }^{1}$ 东南大学, 交通学院, 南京, 中国

${ }^{2}$ 东南大学, 生物科学与医学工程学院, 南京, 中国

${ }^{3}$ 东南大学, 土木工程学院, 南京, 中国

邮箱

213141481@seu. edu. cn（黄少琦）

摘要：为探究在不同营养环境作用下微生物封堵效果的差异。以常水头渗透试验为基础, 向砂土柱中分别添加马铃 薯液、混合营养液 (马铃薯-尿素-氯化铝混合溶液), 定期记录测压管读数, 分析渗透系数变化趋势, 并通过电镜 扫描, 观察土壤微观结构变化。结果表明: 在微生物生长时提供马铃薯液与混合营养液都能得到一定的封堵效果, 添加马铃薯液的砂土柱在 10 天内渗透系数可下降 $83.64 \%$, 添加混合营养液的则可降至 $99.14 \%$, 观察土壤微观结构发 现添加混合营养液的砂粒表面生成了一层明显的膜状胶结物质, 而添加马铃薯液的砂粒表面并无明显改变, 各砂粒 仍呈分散状态。试验表明：微生物封堵技术可以在短期内改善土体性质、实现有效封堵, 且能在一定时间内保持稳 定。 
关键词: 砂土, 微生物诱导碳酸钻沉积, 封堵, 改性

\section{1. 引言}

统计数据表明土木水利工程中发生的事故近 $30 \%$ 都与 渗漏问题有关, 如何快速有效地解决渗漏问题, 从而保证 构造物的安全性、稳定性, 是摆在土木工作者面前的一大 难题。传统的封堵方法, 如水泥灌浆、砌筑防渗墙等, 都 是在人为查找渗漏处的基础上进行封堵处理, 除了经济成 本高, 施工难度大以外, 还存在难以全面探查渗漏位置, 无法对存在渗漏隐患的薄弱处进行预先处理等不足。同时, 由于使用的灌浆材料和固化剂大多含有有毒的化学物质, 极易对环境造成不良影响。相比之下, 基于生物矿化过程 的微生物诱导碳酸钙沉积技术 (MICP) 可将渗漏位置的探 查与封堵结合起来, 经济成本低, 封堵效率高, 环境耐受 性好。

微生物防渗封堵技术主要是利用微生物新陈代谢活 动中产生的尿素酶来对尿素进行分解, 生成碳酸根离子, 然后与环境中的钙离子结合生成碳酸钙晶体沉淀于土壤 颗粒的缝隙内, 以此来实现封堵。由微生物诱导产生的碳 酸钙晶体附着力强, 能将土体颗粒很好地粘结在一起, 同 时还在土体中起填充作用, 可使土体更加密实, 大幅度提 高土体的抗剪强度 [1] [2] [3]。

目前, 在荷兰、日本、加拿大等国都已经开展了对这 一技术的研究并有部分工程应用实例。其中荷兰就利用这 一技术解决了从阿姆斯特丹到巴黎的高速铁路路堤与渡 槽和运河交汇处出现的砂砾岩土层的渗漏问题 [4]。而国 内对于这一技术的研究, 尚处于初级阶段, 且多是以混凝 土作为研究对象。

本研究以砂土为研究对象, 选用粒径较为均匀的标准 砂进行试验。

在营养液选取方面, 由于日本与荷兰的研究人员已成 功利用淀粉副产物Nutrolase制成的营养液使日本砂土与 荷兰砂土实现了封堵 [4], 国内也有选用马铃薯液作为营 养液进行封堵的研究 [5], 因此选取马铃薯液作为第一种 营养液。马铃薯液可提供微生物生长的基本营养物质, 但 无法提供钙离子形成碳酸钻沉淀, 因此选取由马铃薯一尿 素-氯化钙以一定比例混合而成的溶液作为第二种营养液, 进行对比试验。

而对于微生物的来源, 遍观各个试验也多有不同。主 要可分为两类: 一类是直接利用土中原有的微生物, 比如 张贺超 [4]、贾强 [6]的试验; 另一类是向土体中人为添加 微生物, 如钱春香选取的菌种是 “从高碱性盐湖土壤中提 取出来的嗜碱芽孢杆菌” [4], 此外也有选用巴氏芽狍杆 菌或芽狍八叠球菌的试验 [1] [8] [9]。本研究选用的外源 微生物为巴氏芽狍八叠球菌。

具体研究内容包括: 预备试验一得出最适混合营养液 浓度、正式试验一探究微生物封堵效果在马铃薯液和混合 营养液各自作用下的差异、耐久性试验一探究微生物封堵 效果的持久性、电镜分析一观察封堵完成后土壤微观结构 的变化。

\section{2. 试验}

2.1. 试验材料、装置

\subsection{1. 砂土}

试验用砂为厦门ISO标准砂, 将其按渗透试验要求装 入自制常水头渗透试验仪中, 制成直径 $10 \mathrm{~cm}$, 高 $35.5 \mathrm{~cm}$ 的土柱。土柱下垫透水石 (直径为 $10 \mathrm{~cm}$ ) 与快速透水型滤 纸, 上覆金属网与粗砂, 所维持的常水头为 $3 \mathrm{~cm}$ 。

\subsection{2. 营养液}

试验所用营养液主要有两种: 纯马铃薯液与混合营养 液。混合营养液由马铃薯液、尿素、氯化钲按一定比例混 合而成。其中马铃薯液含有微生物生长所必须的基本营养 物质蛋白质和淀粉; 尿素可被微生物中腿酶分解, 生成碳 酸根离子; 氯化钻可提供钙离子, 便于生成碳酸钻沉淀。 马铃薯液由马铃薯粉兑水调和而成, 其中马铃薯含量为 $4.4 \mathrm{~g} / \mathrm{L}$ 。混合营养液设有两种不同浓度, 中浓度由 $4.4 \mathrm{~g} / \mathrm{L}$ 的马铃薯, $1.0 \mathrm{~mol} / \mathrm{L}$ 的尿素 (以含氮量为 $46.4 \%$ 的尿素调 配而成）， $1 \mathrm{~mol} / \mathrm{L}$ 的氯化钙（以含量为 $94 \%$ 的氯化钙调配 而成)制成; 高浓度由 $4.4 \mathrm{~g} / \mathrm{L}$ 的马铃薯, $1.5 \mathrm{~mol} / \mathrm{L}$ 的尿素, $1.5 \mathrm{~mol} / \mathrm{L}$ 的氯化钙制成。

\subsection{3. 菌种}

试验所选取的菌种为巴氏芽孢八叠球菌 (Sporosarcina pasteurii), 购买于中国普通微生物菌 种保藏管理中心（CGMCC），编号1.3687。该菌细胞呈球 状或卵圆形, 直径为 $1-2 \mu \mathrm{m}$, 长为 $2-3 \mu \mathrm{m}$, 革兰氏阳性, 具有少数鞭毛运动, 芽孢呈圆形, 直径在 $0.5-1.5 \mu \mathrm{m}$, 化 能异养菌, 能在 $15-37^{\circ} \mathrm{C}$ 生长, 广泛分布于土壤等环境中 [10]。

试验所用的菌液由东南大学生物工程实验室培育。菌 液数量级约为 $10^{8}-10^{12} / \mathrm{mL}$ 。

培育时采用编号 907 培养基, 主要成分为: 蛋白胨 $5 \mathrm{~g} / \mathrm{L}$ 、 肉浸膏 $3 \mathrm{~g} / \mathrm{L}$ 、尿素 $20 \mathrm{~g} / \mathrm{L}$ 、一水硫酸锰 $30 \mathrm{mg} / \mathrm{L}$ 。配置好后 放到电热炉上加热溶解, 将培养基pH调至9（该菌为嗜碱 芽孢杆菌)。使用LDZX-30FBS立式压力蒸汽灭菌器以 $121^{\circ} \mathrm{C}$ 的温度对烧杯、培养㿼、试管、枪头盒等进行 $20 \mathrm{~min}$ 高压 灭菌, 随后放入干燥箱内干燥 $20 \mathrm{~min}$, 将干燥好的上述用 品放入超净工作台内, 进行浇板, 浇板完成后冷却培养血。

随后进行安剖开封并将菌种转接至培养㿼。使用 $75 \%$ 酒精消毒、用火焰加热顶端、滴少量无菌水到顶端使其破 裂、用锉刀或铌子敲下已经破裂的安剖顶端。以无菌吸管 吸取 $0.3-0.5 \mathrm{~m} 1$ 适宜的液体培养基, 滴入安剖内, 轻轻振 荡, 使冻干菌体溶解呈悬浮液, 平板划线在制备好的培养 皿中。将培养血放入 $30^{\circ} \mathrm{C}$ 恒温培育箱培育, 隔天观察。

待培养血中长出纯菌落后将其转接到LB斜面培养基 中, 放入 $30^{\circ} \mathrm{C}$ 恒温培养箱内继续培养。将 $\mathrm{LB}$ 斜面培养基上 的菌落转接到烧瓶内, 培养适量种子液（根据5\%到10\%的 
比例考虑）。将种子液和配制好的培养液以 $5 \%$ 到 $10 \%$ 的比 例在无菌操作台上混合好, 放入 THZ-C恒温振荡器中以 $200 \mathrm{r} / \mathrm{min} 、 30^{\circ} \mathrm{C}$ 的参数来培养 18 到 $22 \mathrm{~h}$ 后即可使用。

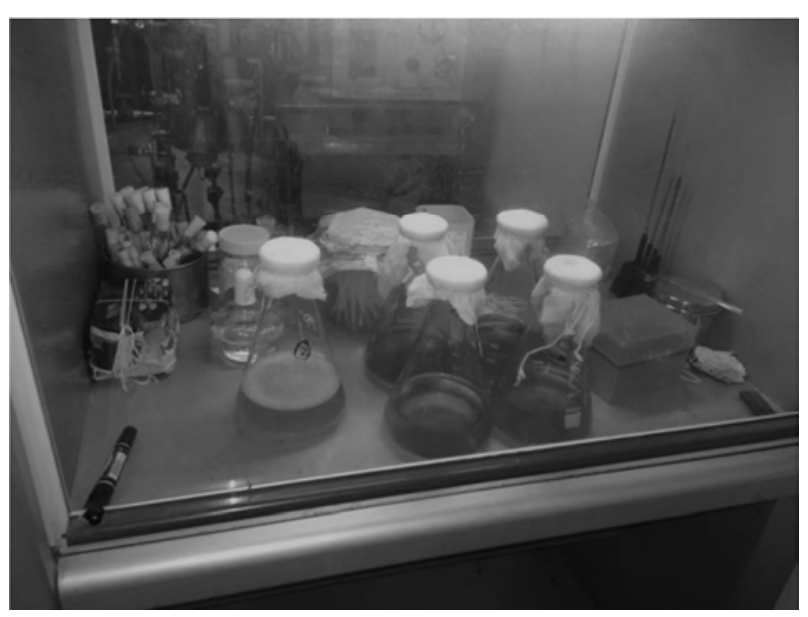

图1 用于扩大化培养的种子液。

\subsection{4. 装置}

试验装置为如图2所示自制常水头渗透仪, 材质分PVC 与有机玻璃两种。装置高 $465 \mathrm{~cm}$, 内径 $10 \mathrm{~cm}$ 。侧面与三个 测压管相连，测压管间隔为 $10 \mathrm{~cm}$ 。顶部入水口与恒温水箱 相连, 由恒温水箱持续通入营养液。为维持常水头在中部 开一中出水口, 便于将高于指定水头的水排出。下部开一 下出水口, 排出废液。

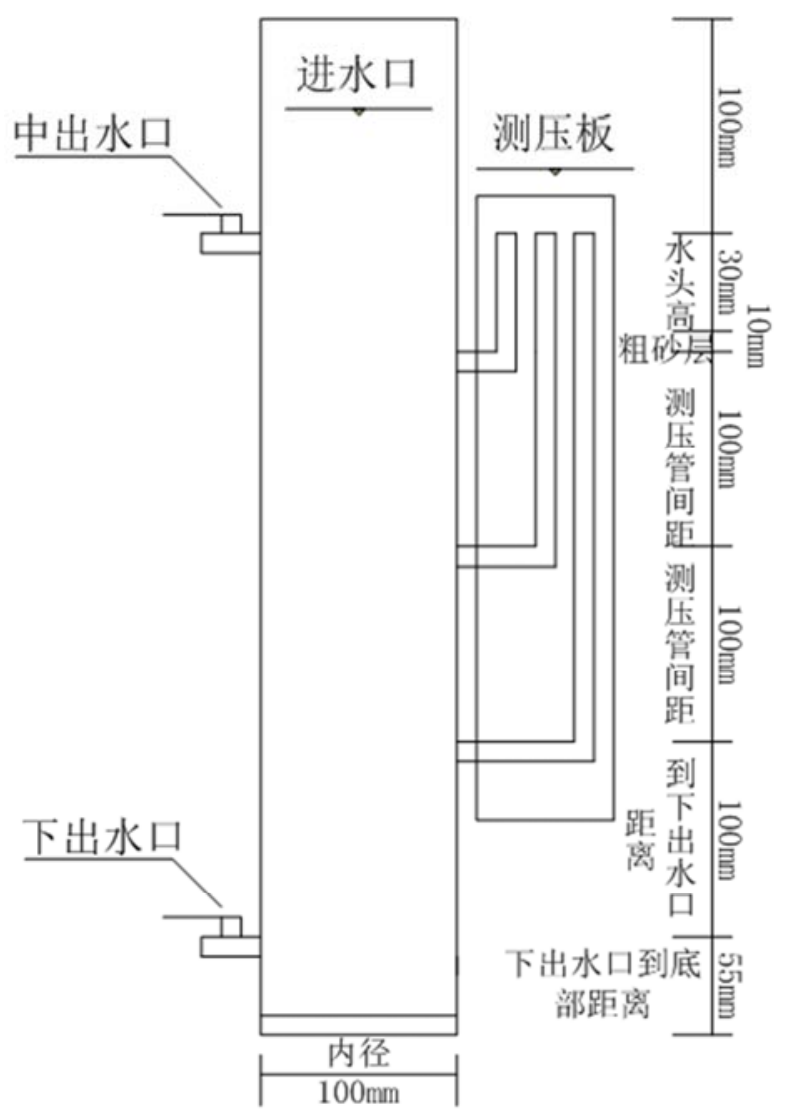

图2 渗透试验装置。

\section{2. 试验步骤}

装配砂土柱, 配置营养液, 将仪器进行搭接; 通入营 养液, 使砂土柱饱和并形成稳定渗流; 如需加入菌液, 则 此时暂停渗流, 由砂土柱上方灌注菌液, 每根土柱添加 800m1菌液, 待菌液完全渗入后, 重新开启渗流; 记录半 小时的流量及测压管水头。试验记录数据的频率为初始两 周每天一记, 之后改为两天一记至无法测出有效数据为止。

砂土柱顶部入水口连接恒温水箱, 由恒温水箱持续通 入温度为 $30^{\circ} \mathrm{C}$ 的营养液 (巴氏芽狍八叠球菌最佳培养温度 为 $30^{\circ} \mathrm{C}$ )。为保证常水头, 多余的营养液由中部出水口排 入一旁的大水箱, 再由泵从大水箱抽回到恒温水箱, 形成 循环。渗流之后的废液由下方出水口汇入自制引流沟渠统 一排出。

\section{3. 试验分组、流程}

预备试验:

已知钙盐浓度超过 $1.0 \mathrm{~mol} / \mathrm{L}$ 时, 微生物中脲酶活性受到 抑制开始下降, 而研究表明在 $1.5 \mathrm{~mol} / \mathrm{L}$ 的浓度以下适量提高 氯化钲和尿素的浓度又可增加碳酸钲的生成量 [11]。为探究 不同氯化钻和尿素的浓度对于渗透系数的影响, 进而选取最 适混合营养液浓度进行后续试验, 因此在有菌情况下设置一 组中浓度和一组高浓度混合营养液的对比试验。

正式试验：（混合营养液浓度选取预备试验中封堵效 果较好的一组)

I 在混合营养液的条件下，设置有菌、无菌的对比试 验, 用以排除菌种以外的因素在混合营养液环境下 对封堵效果的影响。

II 在纯马铃薯液的条件下, 设置有菌、无菌的对比试 验, 用以排除菌种以外的因素在纯马铃薯液环境下 对封堵效果的影响。

III 在有菌情况下, 设置混合营养液与纯马铃薯液的对 比试验, 用以探究不同营养条件下微生物封堵效果 的差异。

表1 正式试验分组情况。

\begin{tabular}{lll}
\hline & 菌种 & 营养液 \\
\hline I & 有无对比 & 最适浓度混合营养液 \\
II & 有无对比 & 纯马铃薯液 \\
III & 有 & 最适浓度混合营养液与纯马铃薯液对比 \\
\hline
\end{tabular}

耐久性试验:

选取已取得一定封堵效果的砂土柱, 将其进行搁置, 观察其后一个月的渗透系数变化, 探究微生物封堵效果的 持久性。

微观结构分析:

通过电镜扫描, 观察在有菌时马铃薯液和混合营养液 两种条件作用下土壤微观结构的变化。

\section{3. 结果与讨论}

由于装填土柱时的操作差异, 包括用木锤击实时的力 道不同以及土柱装好之后搁置时间的差异, 使得各试样初 始渗透系数有一些差异, 但这并不影响我们对其稳定之后 
的渗透系数变化趋势进行分析判断。如图6无菌马铃薯液 条件下渗透系数的变化趋势图, 可见虽然其初始渗透系数 较低, 但稳定后却有所回升, 且无进行进一步封堵的趋势, 符合理论推测。

\section{1. 预备试验}

图3为有菌条件下, 中浓度 $(1.0 \mathrm{~mol} / \mathrm{L})$ 与高浓度 $(1.5 \mathrm{~mol} / \mathrm{L})$ 混合营养液作用下, 渗透系数的变化趋势图。 从图中可看出, 高浓度下微生物的封堵效果相比中浓度的 更加显著快速。第8天时, 高浓度的已经接近完成封堵, 渗透系数平均下降了 $92.5 \%$, 而中浓度的渗透系数平均只 下降了 $65.9 \%$ 。可见虽然钙离子浓度超过 $1.0 \mathrm{~mol} / \mathrm{L}$ 后会对 微生物中脲酶产生抑制作用, 但在 $1.5 \mathrm{mo} / \mathrm{L}$ 的限制浓度之 下, 氯化钙和尿素的浓度越高, 封堵效果也相应越好。因 此后续试验皆采用高浓度混合营养液。

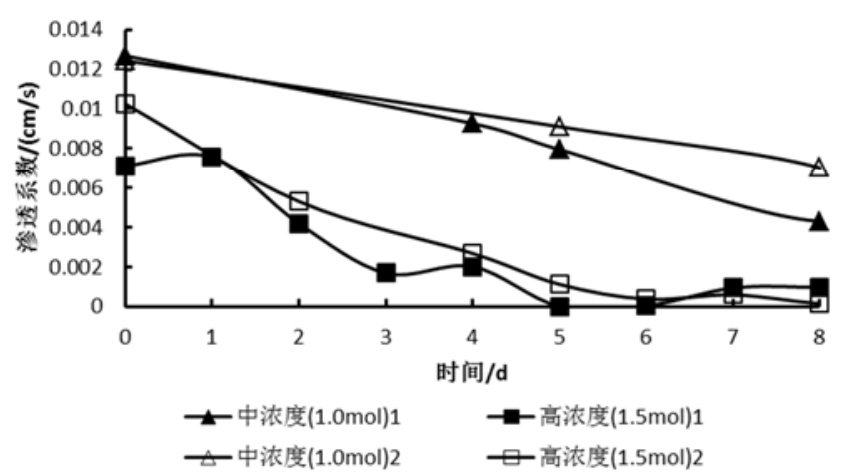

图3 有菌、中浓度与高浓度营养液, 渗透系数变化。

\section{2. 正式试验}

\subsection{1. 混合营养液下有无菌种的对比}

图4为无菌时, 混合营养液作用下渗透系数的变化图。 可以看出渗透系数也有明显的下降, 但由于其初始渗透系 数较小, 实际下降幅度并不大, 且数据波动, 极不稳定, 说明虽有一定封堵效果, 但这种封堵并不是十分稳定有效 的。我们推测这种封堵的成因一方面是由于营养液中的沉 淀, 另一方面我们观察到在无菌时上层土柱会出现大范围 的空洞, 下层土柱较密实, 而有菌的土柱则上下层都较密 实, 因此可能是由于长期的水力冲刷将无菌土柱上层粒径 较小的砂砾带至下层, 由此形成一定的封堵效果。

图5为有菌时, 混合营养液作用下渗透系数的变化图。 可以看出封堵效果是显著且迅速的。在第 5 天大部分试样 的渗透系数就已下降 $92 \%$ 以上。而所有砂土柱的渗透系数 都先后在8-12天之内下降了 $99 \%$, 并在之后依然持续下降。 参考土壤渗透性与渗透系数的对应关系可知, 微生物封堵 使砂土由中渗透性变成了极低渗透性。对比无菌组, 有菌 组封堵更快且能得到更高的封堵率。

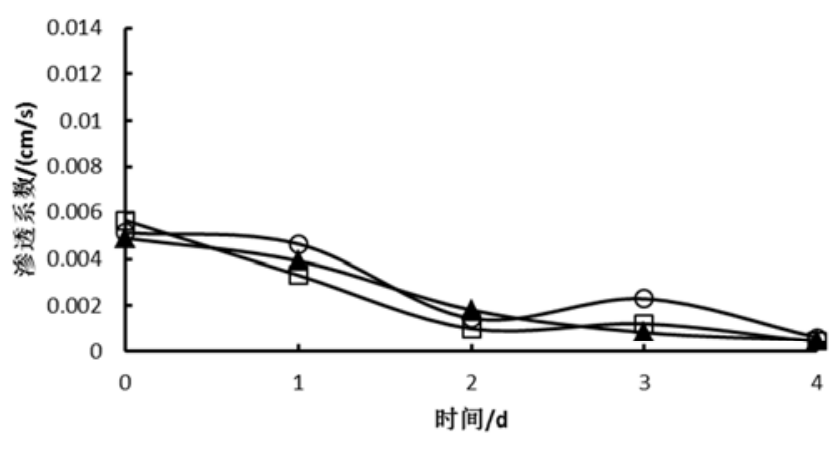

—无菌、营养液1 —无菌、营养液2 —无菌、营养液3

图4 无菌、高浓度营养液, 渗透系数变化。

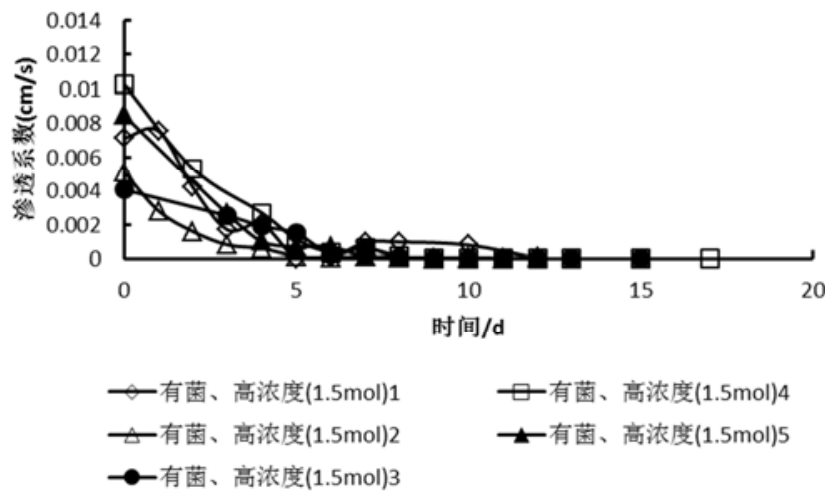

图5 有菌、高浓度营养液, 渗透系数变化。

\subsection{2. 纯马铃薯液下有无菌种的对比}

图6为无菌时, 纯马铃薯液作用下渗透系数的变化图。 可以看出渗透系数基本没有下降, 甚至还有回升。但由于 此三个试样的初始渗透系数就较低, 我们看到稳定之后的 渗透系数基本在 $0.005 \mathrm{~cm} / \mathrm{s}$ 左右, 为中低渗透性, 可知纯 马铃薯液也可以产生一定的封堵。这种封堵的成因同样可 归结于马铃薯液中的沉淀和水力冲刷产生的砂砾迁移作 用。

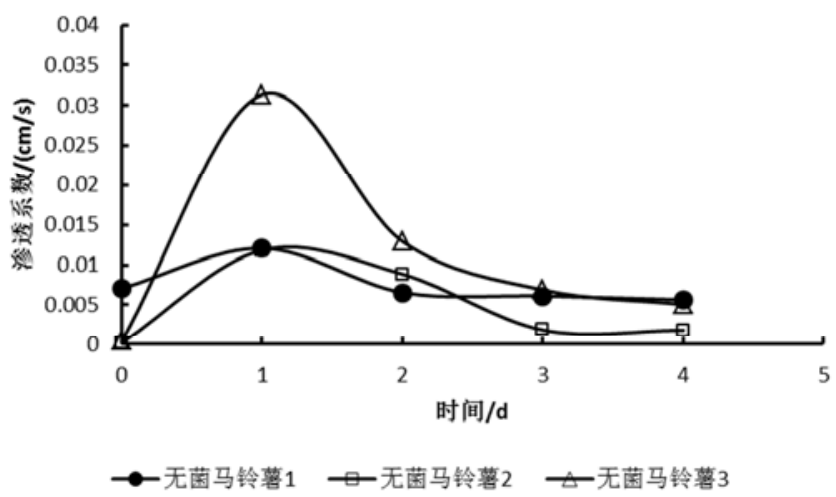

图6 无菌、马铃薯液, 渗透系数变化。

图7为有菌时, 纯马铃薯液作用下渗透系数的变化图。 可以看出渗透系数波动很大, 尤其是初期十分不稳定, 但 仍可以看出总的趋势是变小的。在 15 天后也能达到使渗透 系数下降 $82 \%$ 的效果。可知微生物在纯马铃薯液的环境下 也能实现一定的封堵, 只是所需时间较长且效果不够稳定。 
由于环境中没有尿素和氯化钻, 无法形成碳酸钻沉淀, 但 渗透系数最后几乎都维持在 $0.001 \mathrm{~cm} / \mathrm{s}$, 相比无菌时纯物 理性的封堵, 有菌时的封堵显然效果更加显著, 成因也有 所不同。那么我们可以推测其封堵成因除了马铃薯液中的 沉淀和砂砾迁移, 还可能是由于微生物的繁殖及其产生的 分泌物。

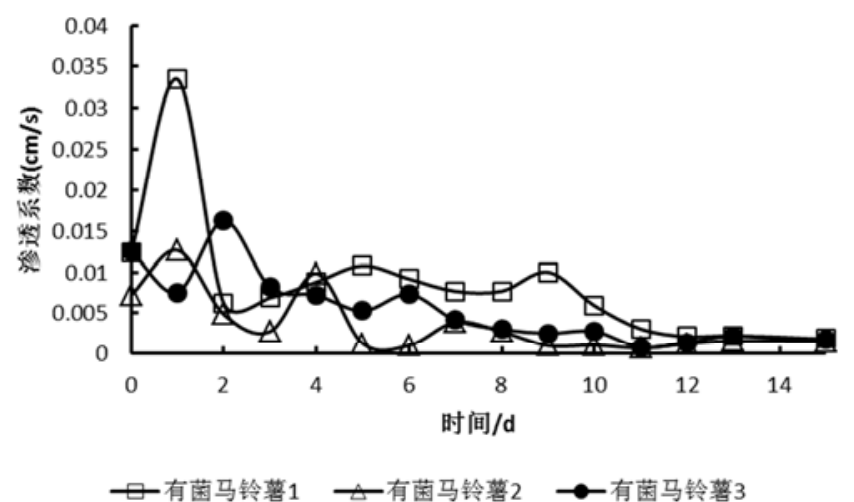

图7 有菌、马铃薯液, 渗透系数变化。

3. 2. 3. 混合营养液与纯马铃薯液下菌种封堵效果的对比

图8为有菌条件下，营养液与纯马铃薯液作用下渗透 系数变化的对比图。

可以看出相比营养液作用下的封堵效果, 单凭马铃薯 液封堵效果极不稳定, 且所需时间较长。但是, 没有尿素 分解后提供碳酸根离子, 没有氯化钲提供钻离子, 在无法 形成碳酸钻的情况下依然实现了一定的封堵效果, 如前文 所述推测可能是由于马铃薯液能够提供微生物生长的基 本营养物质, 虽然无法生成碳酸钙, 但大量繁殖生长的微 生物以及其分泌排泄物等也能起到一定的封堵作用。同时, 由于并没有形成碳酸钙, 无法粘结砂砾堵塞孔隙, 因此这 种封堵是极不稳定的, 体现到数据之上就是渗透系数波动 较大。

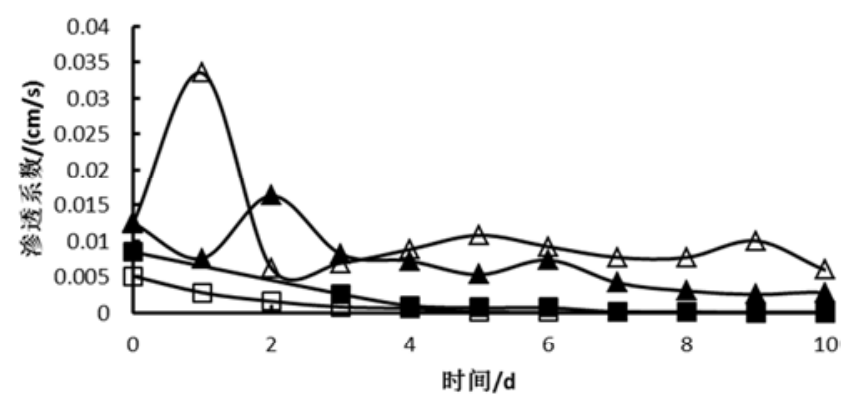

一一有菌、营养液 $(1.5 \mathrm{~mol}) 1$ 一有菌、马铃暮1

—有菌、营养液 $(1.5 \mathrm{~mol}) 2$ —有菌、马铃薯 2

图8 有菌、马铃薯液与高浓度营养液, 渗透系数变化。

\section{3. 封堵效果耐久性试验}

图9为一土柱在形成一定程度的封堵（渗透系数下降 60\%）之后，将其搁置一旁不再通入任何液体，一个月内 渗透系数的后续变化趋势图 (图中原始渗透系数为该土柱 注入微生物进行封堵之前的渗透系数)。可以看出搁置试
样渗透系数略有回升, 但回升之后相比改土柱初始渗透系 数仍具有很明显的封堵效果。这表明微生物诱导封堵具有 一定的耐久性。

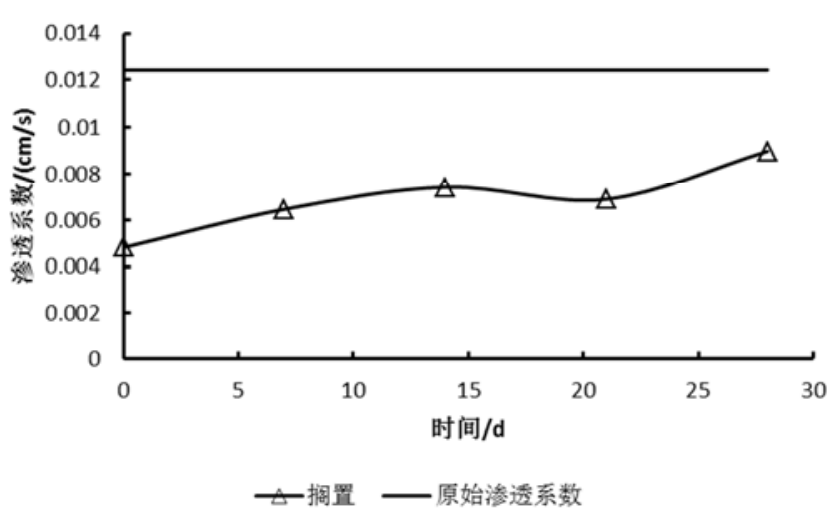

图9 被搁置试样的渗透系数变化。

\section{4. 土体微观结构变化检验}

\section{4. 1 . 纯马铃薯液}

在土柱上、中、下部分别取一土样, 放入烘箱以 $110^{\circ} \mathrm{C}$ 高温烘干 6 小时, 用场发射扫描电子显微镜进行砂粒表面 结构观察, 发现砂粒表面并无明显变化。

\section{4. 2. 混合营养液}

将封堵系数降低 $99 \%$ 的土柱取样以电镜扫描后如图 10 ，可见砂粒表面形成了一层明显的膜状物质，可将砂 粒粘结起来, 能谱分析如表 2 , 发现主要元素为 $\mathrm{C}$ 以及Ca。 土柱中的 $\mathrm{Ca}$ 元素和 $\mathrm{Cl}$ 元素绝大多数来自 $\mathrm{CaCl}_{2}$, 由检测所 得的 $\mathrm{Cl}$ 和 $\mathrm{Ca}$ 原子数百分含量比并非 $2: 1$, 可以推测 $\mathrm{Ca}$ 元素 以其他化合物的形式, 即生成碳酸钙沉淀, 留在土柱中。

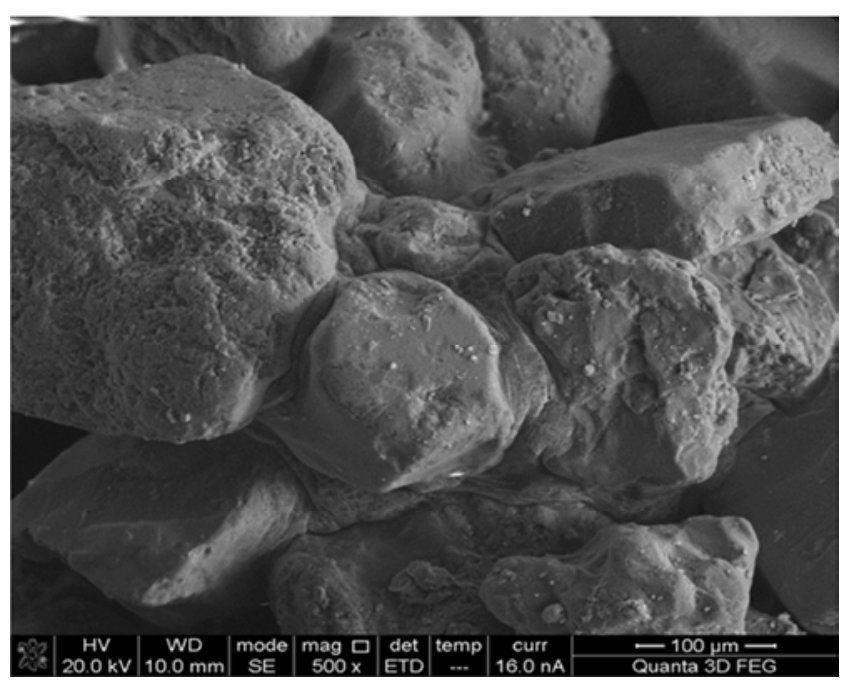

图10 有菌、营养液作用后，渗透系数下降 $99 \%$ 的砂粒在电镜下的微观结 构。 


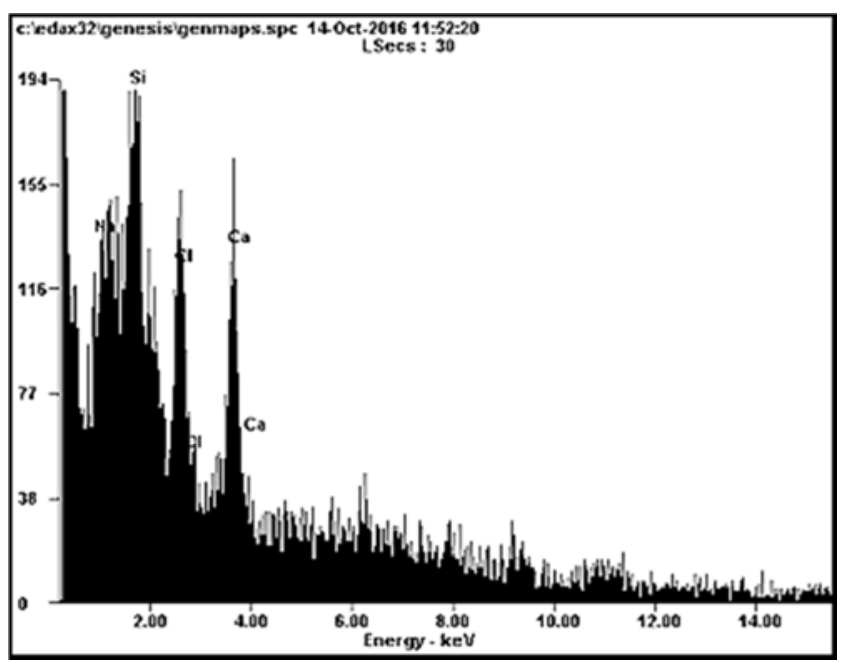

图11 对膜状物质进行的电镜能谱分析图。

表2 电镜能谱分析元素组成表。

\begin{tabular}{lll}
\hline Element & Wt\% & At\% \\
\hline NaK & 14.99 & 21.46 \\
SiK & 16.55 & 19.40 \\
C1K & 27.18 & 25.24 \\
CaK & 41.28 & 33.90 \\
Matrix & Correction & ZAF \\
\hline
\end{tabular}

\section{4. 结论}

本文通过实验探究微生物在砂土中的封堵效果, 主要 可得出以下结论: 无菌状况下，单凭通入纯马铃薯液或混 合营养液都只能实现一定的封堵, 这种封堵的封堵率较小 且封堵效果较不稳定。用纯马铃薯液作为微生物生长的营 养液可以实现封堵，15天后渗透系数可下降 $82 \%$, 但封堵 速率仍不及高浓度混合营养液且同样存在封堵效果不够 稳定的问题。用混合营养液作为微生物生长的营养液可以 成功进行封堵, 且封堵速率随着营养液浓度的增大而增大, 其中高浓度营养液封堵效果尤为显著, 12 天后渗透系数即 下降 $99 \%$, 封堵效果较为稳定, 渗透系数波动较小。停止 添加营养液之后渗透系数会略有回升, 但回升之后仍具有 很明显的封堵效果, 说明微生物封堵有一定持久性。而电 镜扫描结果显示砂粒表面形成了一层明显的膜状物质, 将 砂粒粘结起来。在本次试验中并未特意设置裂缝或渗漏孔, 而是依靠微生物自身去寻找土壤中的细小孔隙并进行封 堵补强从而使渗透系数减少进而达到防渗效果, 说明微生 物封堵技术能有效将渗漏处的探查与封堵结合起来。

相比传统的封堵方式, 微生物封堵的优势在于其体积 小, 易于进入裂缝, 形成深度封堵。同时流动性较大, 可
随营养物质优先进入大裂隙进行选择性封堵。虽然目前已 有成功的现场试验, 但若要将其作为一项技术在现场进行 规模化应用, 还有一些值得探究的问题。如营养液的选取, 封堵位置的控制和封堵维持的时间等。适宜微生物生长的 营养液较多，但如果用于大规模封堵应用，除了成本问 题, 理论上不希望营养液具有粘度, 这样才能随注入水优 先进入高渗透带, 实现选择性封堵。为实现设计位置封堵, 可将微生物与营养液分开注入, 以营养液诱导微生物进行 封堵, 但微生物生长与营养消耗速度无法预测, 且封堵效 果也与所处环境直接相关, 如何使其持续有效地对指定位 置进行封堵尚还需要进一步研究。而目前虽证实了微生物 封堵切实有效, 但对于其封堵作用究竟能维持多长时间并 不清楚，这也须在现场试验过程中慢慢摸索。

\section{参考文献}

[1] 赵茜. 微生物诱导碳酸钙沉淀MICP固化土壤实验研 究. [D]. 北京: 中国地质大学. 2014, 5。

[2] 姜欢. 微生物诱导碳酸钻沉积防渗堵漏的试验研究. [D]. 济 南: 山东建筑大学. 2014。

［3］沈春林. 防水堵漏工程技术手册. [M] . 北京：中国建材工 业出版社．2010。

４4］张贺超, 郭红仙, 李萌, 等. 砂土介质中微生物诱导封堵 试验研究. [J]. 工业建筑. 2015 (1) : 139-142。

[5] 谭谦, 郭红仙, 程晓辉. 微生物水泥砂浆的强度及耐久性 试验研究. [J]. 工业建筑. 2015 (7) : 42-47。

［6］贾强, 杨金彪, 张金金, 等. 土壤中微生物防渗封堵技术的 试验研究. [N]. 建筑材料学报. 2014, 17 (4)。

[7]钱春香, 李瑞阳, 等.混凝土裂缝的微生物自修复效 果. [N]. 东南大学学报. 2013, 43(2)。

[8] 贾强, 张金金, 侯宏涛, 等. 微生物沉积碳酸钻修复混凝土 裂缝的现场试验. [N]. 建筑材料学报. 2013, 16(4)。

[9] 张君洁, 苗晨曦. 微生物固化砂土实验研究. [D]. 武汉: 华中科技大学。

[10］杨钻. 高强微生物砂浆机理与工作性能研究. [D]. 北京: 清华大学 (土木工程).2013, 4。

[11] 张越. 微生物用于砂土胶凝和混凝土裂缝修复的试验研 究. [D]. 北京: 清华大学 (土木工程).2014, 6 。 